\title{
Small-Angle Neutron Scattering Study of the Relaxation of a Melt of Polybutadiene H-Polymers Following a Large Step Strain
}

\author{
M. Heinrich, ${ }^{*, \dagger}$ W. Pyckhout-Hintzen, ${ }^{*, \dagger}$ J . Allgaier, ${ }^{\dagger}$ D. Richter, ${ }^{\dagger}$ E. Straube, ${ }^{\ddagger}$ \\ T. C. B. McLeish,§ A. Wiedenmann," R. J . Blackwell, ${ }^{\perp}$ and D. J . Read*,\# \\ Forschungszentrum J ülich, Institut für F estkörperforschung, Postfach 1913, D-52425 J ülich, Germany; \\ Martin-Luther-Universität Halle-Wittenberg, Fachbereich Physik, D-06099 Halle, Germany; \\ Department of Physics and Astronomy, University of Leeds, Leeds, LS2 9J T, U.K.; Hahn-Meitner \\ Institut, D-14091 Berlin, Germany; Department of Applied Maths and Theoretical Physics, Centre for \\ Mathematical Sciences, Wilberforce Road, Cambridge, CB3 OWA, U.K.; and Department of Applied \\ Mathematics, University of Leeds, Leeds, LS2 9J T, U.K.
}

Received August 21, 2003

\begin{abstract}
We present scattering data from a melt of $\mathrm{H}$-shaped polybutadiene polymers, in which the central crossbar of the polymers is wholly labeled with deuterium. The melt was uniaxially stretched by a factor of 2, and using careful temperature control of the sample, the neutron scattering pattern was measured after several different times of relaxation. The scattering data are compared against the predictions of a new theory, based upon the tube model but which includes the additional effect of elastic inhomogeneities in the entanglement network. We find that with this new theory one can obtain a good representation of the data, using parameters that are consistent with relaxation times obtained from a tube model description of the linear rheological data. The most important aspect of these data is an increasing anisotropy of the correlation hole peak, and this appears to be directly related to the elastic inhomogeneities. One further aspect of the comparison to theory is that the best fits are obtained using a Warner-Edwards tube diameter that deforms in a manner consistent with recent predictions for polymer networks.
\end{abstract}

\section{Introduction}

The tube model ${ }^{1}$ is, without doubt, the most successful current theoretical framework for understanding the rheological properties of entangled polymer melts. In its early stages of development, it gave a good qualitative description, with some quantitative successes such as prediction of the "damping function" of linear polymer mel ts after large step strains. The past decade or so has witnessed several important advances with the model, notably resolving a number of known discrepancies between the original model and experiment, for example predicting the observed $M^{3,4}$ power law for melt viscosity as a function of molecular weight, ${ }^{2-4}$ and the use of "convective constraint release" to el imi nate the prediction of catastrophic shear thinning present in the original Doi-E dwards model..$^{5-8}$ One particular advantage of the molecularly based tube model is that it predicts the rheological response of the polymer melt by describing the distribution of chain conformations in the flowing or deformed state. This opens up the possibility that techniques such as small-angle neutron scattering (SANS), which are sensitive to the overall chain conformation, can be used as an independent check on the predictions of the tube model.

In fact, such neutron scattering experiments on melts of linear polymers go back as far as the early progress made by Boué and co-workers, ${ }^{9-13}$ who studied relaxation after a step uniaxial deformation of polystyrene melts. In their initial experiments, the labeled and

$\dagger$ Institut für Festkörperforschung.

₹ Martin-Luther-Universität Halle-Wittenberg.

$\S$ Department of Physics and Astronomy, University of Leeds.

" Hahn-Meitner Institut.

$\perp$ Centre for Mathematical Sciences.

\# Department of Applied Mathematics, University of Leeds. unlabeled linear chains had similar molecular weight, which meant that they were measuring, as directly as possible, the single-chain scattering function.9,10 They were hoping, but unable, to detect a signature in the scattering of the predicted chain retraction along the tube contour. ${ }^{1}$ Subsequent experiments by the same group studied the relaxation after step deformation of both asymmetric melts (with long labeled chains and short unlabeled ones) and networks swollen by a labeled solvent. ${ }^{11-13}$ In each case "butterfly" patterns were seen on time scales greater than the relaxation time of the short chains or solvent. The generally accepted explanation for these "butterfly" patterns does not specifically invoke the tube model. Elastic fluctuations and inhomogeneities in the entanglement network of the long polymers, or in the chemically bonded network, are supposed to couple to the motion of the short chains or solvent. ${ }^{14}$ The result is enhanced scattering in directions parallel to the stretch, which is the main feature of these patterns. One aspect of this is that the scattering parallel to the stretch is apparently enhanced down to the zero wavevector limit because the elastic inhomogeneities are present even on large length scales and the mobile short chains or solvent can move large distances in response to these.

More recent experiments on linear polymer chains have returned to use of melts where the labeled and unlabel ed linear chains have similar molecular weight, and one can be reasonably sure of measuring the single chain scattering. One such experiment ${ }^{15}$ has revisited relaxation after step strain, using polyisoprene melts where the tube diameter is significantly smaller than for polystyrene (but where the number of entanglements per chain is approximately the same as in the longest polymers used by Boué). Another experiment ${ }^{16}$ has studied the scattering at various points within a steady- 
state flow in a complex geometry (contraction flow). In this experiment, each polymer experiences a flow history which depends on its particular path through the flow cell, so the experiment constitutes a strong test of a molecularly based rheological theory.

In the context of long-chain branched polymer melts, the tube model has also had a degree of success. A molecular theory for rather generic topologies of LCB polymers was derived from the physics of the tube model..$^{17}$ It explained successfully how the melts can be strongly strain hardening in both planar and uniaxial flows but strain softening in shear flows. Recently a "multimode" version of the theory has been used to account quantitatively for a very wide set of nonlinear rheological data on a standard LDPE. 18,19 To achieve this, it was necessary to invoke a physical consequence of the tube model around the branch points themselves. Termed "branch point withdrawal", it describes the shift of a branch point within a LCB polymer under strain toward the tube containing the strand of greatest tension meeting at the branch point.20,21 To test this theory, a recent set of scattering experiments ${ }^{22,23}$ have focused on $\mathrm{H}$-shaped polymers, the simplest architecture containing the minimum two branch points required to exhibit nonlinear deformation behavior substantially different from that of linear polymer chains. These experiments take advantage of advances in controlled polymer synthesis, using "block copolymer" chains that are partially label ed with deuterium. In the case of the $\mathrm{H}$-shaped polyisoprene used in refs 22 and 23, the tips of the polymer arms were deuterium-labeled. This particular labeling was designed with a view toward detecting the specific mechanism of branchpoint withdrawal. It had been conjectured ${ }^{24}$ that the localization of labeled material produced when the arms were pulled into the same tube segment should produce an increased scattering. In common with the early experiments of Boué and co-workers, these experiments examined the relaxation of the polymer melt foll owing a uniaxial step strain.

The modeling of such experiments required the development of a detailed random phase approximation (RPA) theory of polymer melts for constraints held out of equilibrium. ${ }^{25}$ Despite using, as closely as possible, the tube model description of the chain deformation, this theory did not accurately describe the experimental $\mathrm{H}$-polyisoprene data. While there were indi cations that including effects such as a partial branchpoint withdrawal improved the fit, the fact remained that there were aspects of the experimental data which were not correctly described. These discrepancies were most evident at the longest relaxation times probed in the experiments, when one would expect most of the $\mathrm{H}$ polymer arms to have escaped their original tube and relaxed their orientation. In this regime, the intensity of the apparent "correlation hole" peak parallel to the stretch direction was significantly greater than at shorter relaxation times, while the perpendicular peak remained at approximately the same magnitude. This increase in anisotropy occurred despite the fact that the stress was relaxing. Although the theory could, to some extent, reproduce this effect qualitatively, the magnitude of the anisotropy defied explanation.

A number of possible mechanisms, which qualitatively accounted for this discrepancy between theory and experiment, were suggested. ${ }^{23}$ The accompanying theoretical paper ${ }^{26}$ provides a detailed investigation of one of these mechanisms. It was conjectured that the same elastic fluctuations and inhomogeneities giving rise to the butterfly patterns in the experiments of Boué et al. could have an effect on the scattering in these $\mathrm{H}$ polymer experiments. While there are no free chains or solvent in the $\mathrm{H}$-polymer melt, it seemed possible that the arms of the $\mathrm{H}$-polymer could act as an effective "solvent" and that they could move far enough (a distance on the order of their radius of gyration) to produce enhanced scattering at finite wavevectors. The accompanying paper predicts that this mechanism does indeed occur and that it significantly alters the predicted scattering from stretched $\mathrm{H}$-polymer melts. Using a particular model for the tube, it provides a "first principles" prediction of the effect.

To provide further information on the deformation processes in $\mathrm{H}$-shaped polymer melts, this paper will present scattering data from a polybutadiene melt with a different labeling to the polyisoprene melts of the previous experiments. In this case, the "crossbar" of the $\mathrm{H}$-polymer is wholly labeled with deuterium, and the arms of the polymer are unlabeled. We shall provide detailed comparison with results from the new theory. Our previous publication on the polyisoprene sampl $\mathrm{e}^{23}$ gave full details of the experimental procedure and described in some detail the expected relaxation processes from a tube model description of $\mathrm{H}$-polymers foll owing a large step strain. For these details, we refer the reader to that publication; we shall discuss them only in outline in the following text.

\section{Experimental Section}

2.1. Sample Preparation. The $\mathrm{H}$-polymer of polybutadiene $43 \%$ 1,2-content was prepared by reaction of living anionic arms with a previously made deuterated difunctional crossbar to yield to a large extent the 4-armed branched structure as described earlier. ${ }^{27}$ The microstructure was achieved by the addition of a given amount of triethylamine to the apolar solvent. This was kept constant in the synthesis of both constituents. By fractionation most of the lower and higher functionality products could be eliminated, but temperature gradient interaction chromatography (TGIC) investigations showed that the final product still contains, in addition to the expected $\mathrm{H}$-polymer, 25 wt \% of lower branching degrees $(\mathrm{H}$ polymer with missing arm(s)) and $5 \%$ of higher branched products (branched polymers with double crossbar). ${ }^{27}$ Average molecular weights for the arm and the crossbar were determined by membrane osmometry (MO) and size exclusion chromatography (SEC) to be respectively $M_{n, a}=25000 \mathrm{~g} \mathrm{~mol}^{-1}$ and $M_{n, b}=57200 \mathrm{~g} \mathrm{~mol}^{-1}$. The sample was mixed to $0.1 \%$ with the antioxidant BHT to avoid chemical degradation or oxidation upon handling. Strips of length $50 \mathrm{~mm}$, width $10 \mathrm{~mm}$, and thickness $1 \mathrm{~mm}$ were obtained from vacuum-molding at $\mathrm{T}=55^{\circ} \mathrm{C}$ and gentle pressure ( 0.5 bar) for approximately 5 h.

2.2. Dielectric Spectroscopy. The glass transition temperature of the polymer was determined from the dielectric loss maximum at $10^{-3} \mathrm{~Hz}$ (BDS6000, Novocontrol) to be -69 $\pm 2{ }^{\circ} \mathrm{C}$, which agrees to within $1{ }^{\circ} \mathrm{C}$ with DSC measurements of linear analogues of the same microstructure. Differences between the microstructures in arms and crossbar are averaged over

2.3. Rheology. Dynamic mechanical experiments in oscillatory linear shear were performed on a rheometer Ares (Rheometric Sci.) in the parallel plate geometry with diameter $25 \mathrm{~mm}$, at 10 temperatures regularly spaced between +100 and $-35^{\circ} \mathrm{C}$ and frequencies between 0.01 and $100 \mathrm{rad} / \mathrm{s}$. The strain amplitude was $2 \%$ to ensure linearity in the full temperature range. SEC experiments performed on the sample afterward showed that no thermal chain degradation or detectable cross-linking occurred during the rheological experi- 


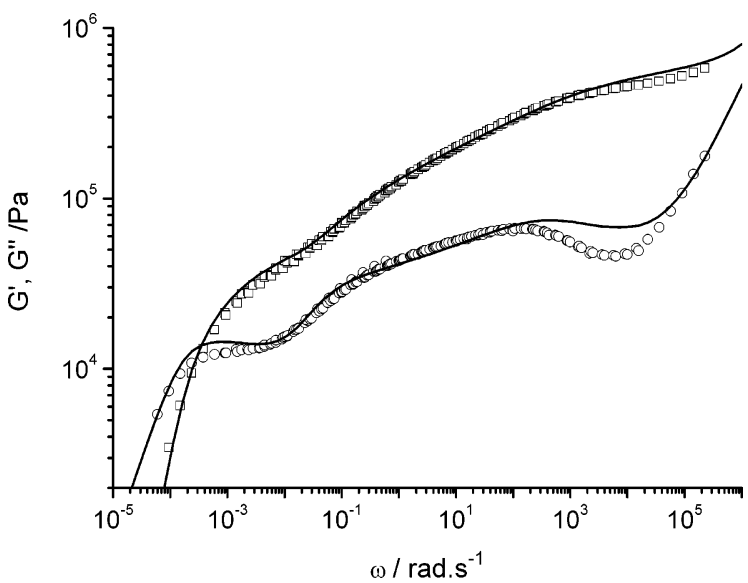

Figure 1. Experimental dynamic moduli G' (squares) and G" (circles) in oscillatory shear at $25^{\circ} \mathrm{C}$ compared with a theoretical fit from a tube model calculation.

ments. The obtained dynamic moduli $\mathrm{G}^{\prime}(\omega)$ and $\mathrm{G}^{\prime \prime}(\omega)$ were shifted to a reference temperature of $\mathrm{T}_{0}=25^{\circ} \mathrm{C}$, using the WLF time-temperature superposition principle..28 The master curves are shown in Figure 1. It was found that the branched $\mathrm{H}$-polymer behaved in a rheologically simple fashion, obeying WLF with small deviations apparent only in the low-frequency regime. (These are not considered to be relevant to the present SANS experiments, which probe relaxations in the higher frequency range only.) The corresponding WLF coefficients were $C_{1}=7.17$ and $C_{2}=189.2$ at $T_{0}$, in reasonable agreement with related microstructures. Arm and crossbar relaxation processes show up as well-separated humps in the loss modulus at high and low frequencies, respectively, as has been described previously. ${ }^{22,23}$

Figure 1 also shows theoretical predictions for the dynamic moduli obtained using the calculation described in ref 22. The data are reasonably well modeled by the theory, except perhaps in the transition region between the high-frequency "Rouse relaxation" regime and the beginning of the broad peak in G" correponding to the arm relaxation. The corresponding fitting parameters are $\mathrm{M}_{\mathrm{e}}=2000 \mathrm{~g} \mathrm{~mol}^{-1}, \mathrm{G}_{0}=0.6 \mathrm{MPa}$, and $\tau_{\mathrm{e}}=6.5 \times 10^{-7} \mathrm{~s}$, which yields $\mathrm{M}_{\mathrm{a}} / \mathrm{M}_{\mathrm{e}}=13$ and $\mathrm{M}_{\mathrm{b}} / \mathrm{M}_{\mathrm{e}}=29 . \mathrm{In}$ obtaining this fit, we have treated $\mathrm{M}_{e}$ and $\mathrm{G}_{0}$ as independent parameters. Although these should in principle be related via $\mathrm{G}_{0}=\rho \mathrm{RT} / \mathrm{M}_{\mathrm{e}}$, the chosen parameters do not exactly satisfy this relationship. (The value of $\mathrm{M}_{\mathrm{e}}$ used is consistent with a plateau modulus of roughly 1.1 MPa.) For comparison, the plateau modulus for linear unlabel ed PB of the same 43\% 1,2microstructure according to ref 29 is $\mathrm{G}_{0}=0.8 \mathrm{MPa}$ at $298 \mathrm{~K}$. We attribute this discrepancy and the fair but not perfect fit to the curve shapes as being due to the significant fraction of impurities in the sample, as discussed above. These are not accounted for in the rheological fit. In view of these impurities, the theoretical description of the rheological data is of reasonable quality and allows us to estimate the expected fraction, $x$, of the arms that relax and escape from the oriented tube as a function of the annealing time following a step strain in the linear regime. ${ }^{22}$ This estimate is shown in Figure 2 . We shall use this relaxation spectrum as a basis for estimating the expected fraction of arm that should relax as a function of annealing time following the nonlinear step strain of the SANS experiments. Of course, it is not clear that this relaxation spectrum from the linear rheology should be appropriate in the nonlinear regime, but the relaxation mechanisms are likely to be similar, and the linear relaxation spectrum should provide at least an initial point for comparison.

2.4. Strain Rig. A strain rig with precise temperature as well as strain rate control was built at FZJ ülich, allowing the straining in situ in the neutron beam for low- $T_{g}$ materials. The sample is fixed vertically in two clamps inside a neutron transparent quartz cell. The lower cylinder is static whereas the upper piston may be driven up by a step motor ensuring a constant strain rate (between $4 \times 10^{-7}$ and $4 \mathrm{~s}^{-1}$ ) during the

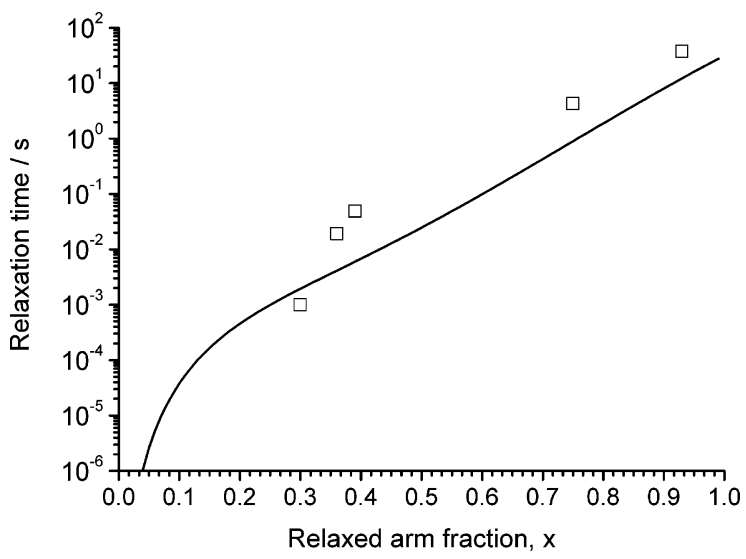

Figure 2. Theoretical annealing time as a function of $x$, the fraction of the arms that have relaxed, as obtained from the fit to linear rheology data given in Figure 1. Also shown are the values of $x$ used to fit the scattering data at each relaxation time.

deformation. The constant strain rate condition is fulfilled by exponentially increasing the crosshead speed as $v(\mathrm{t})=\dot{\epsilon} \mathrm{L}_{0}$ exp$(\dot{\epsilon} \cdot \mathrm{t})$, corresponding to an increase in the sample length as $\mathrm{L}(\mathrm{t})$ $=\mathrm{L}_{0} \exp (\dot{\epsilon} \cdot \mathrm{t})$. The temperature can be varied between -140 and $+200^{\circ} \mathrm{C}$ with an accuracy of $\pm 0.1^{\circ} \mathrm{C}$ by means of gaseous $\mathrm{N}_{2}$ flowing in the quartz cell and controlled by four thermocouples ( $\mathrm{Fe}-\mathrm{Cu}$ ) measuring the temperature in the immediate vicinity of the sample. More details concerning this experimental device can be found in ref 23.

2.5. Scattering. The SANS experiments have been performed at the instrument KWS1 in FZ J ülich at a neutron wavel ength $\lambda_{N}=7 \AA$ with relative dispersion $\Delta \lambda_{N} / \lambda_{N}=20 \%$ and at the instrument V4 in $\mathrm{HMI}$ Berlin at $\lambda_{\mathrm{N}}=8 \AA$ with relative dispersion $\Delta \lambda_{N} / \lambda_{N}=11 \%$. In each case, two sampledetector distances ( 2 and $8 \mathrm{~m}$ at KWS1 and 2 and $7 \mathrm{~m}$ at V4) have been used to cover the scattering vector range $7 \times 10^{-3} \AA^{-1}<\mathrm{q}<1.2 \times 10^{-1} \AA^{-1}$, the scattering vector being defined from the scattering angle $\theta$ as $q=4 \pi \sin (\theta / 2) / \lambda_{N}$. The 2-dimensional macroscopic differential scattering cross sections were obtained from the scattered intensity after correction pixel by pixel for the sensitivity of the detector, subtraction of empty cell scattering, dark current, and absolute calibration by means of lupolene or water standards. The data along the axis parallel and perpendicular to the deformation were obtained from a procedure that eliminates systematic errors upon regrouping angularly, as described earlier. ${ }^{30}$

The sample was initially stretched to a strain $\lambda=2$ at $\mathrm{T}=$ $-40{ }^{\circ} \mathrm{C}$ in $5 \mathrm{~s}$, at a constant strain rate $\dot{\epsilon}=1.386 \times 10^{-1} \mathrm{~s}^{-1}$, and then quenched to $-85{ }^{\circ} \mathrm{C}$ within about $3-5 \mathrm{~s}$. As the typical time for such a scattering experiment is around $7 \mathrm{~h}$, the sample needs to be quenched before each SANS experiment in order to freeze in the sample conformation and avoid uncontrolled relaxation processes. After the scattering experiment, relaxation processes can be restarted by increasing the sample temperature above $\mathrm{T}_{\mathrm{g}}$ and allow measurements of scattering functions at higher annealing times. The temperature history of the sample is given in Figure 3. It has been checked on test samples (polystyrene melts and polyisoprene networks) that the deformation is homogeneous, giving some confidence that the temperature distribution is also homoge neous within the samples. The effective relaxation times at $25{ }^{\circ} \mathrm{C}$ (taking into account the full temperature history, including the heating ramps) are determined using the WLF time-temperature shift factors given in section 2.3. They are known to the extent that WLF is applicable, assuming that the sample instantaneously assumes the gas temperature and that the accuracy of the WLF shift factors is sufficiently good that the extrapolation over 5-6 decades in time is wellbehaved. Reasonable error bars are $100 \%$ in time, which are equivalent to an error in the relaxed arm fraction of $\Delta x \sim 0.1$. Because of the form of the heat/cool cycle, we believe that the temperature of the sample is more likely to slightly below that 


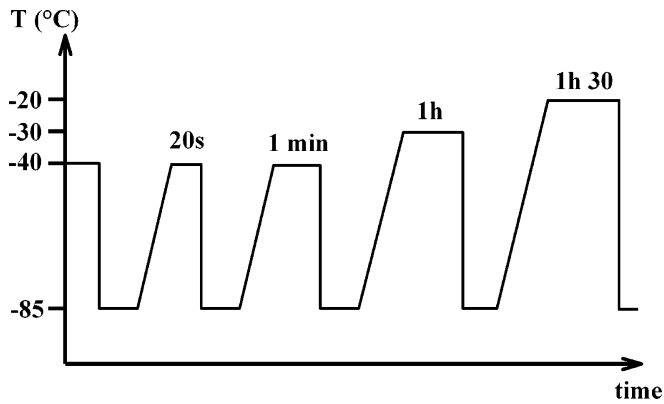

Figure 3. Temperature history of the sample, after a strain at $-40{ }^{\circ} \mathrm{C}$ to $\lambda=2$. SANS experiments are performed at -85 ${ }^{\circ} \mathrm{C}$ after annealing times equivalent to " 0 ", $1.9 \times 10^{-2}, 4.9 \times$ $10^{-2}, 4.3$, and $37.2 \mathrm{~s}$ at $25^{\circ} \mathrm{C}$.

of its surroundings than above it, and for this reason the estimated fractions $x$ should be considered always to be too high.

\section{Parameters in the Theoretical Model}

The RPA calculation of scattering from a stretched copolymer melt, including both the effects of tube constraints held out of equilibrium and elastic fluctuations and inhomogeneities in the entanglement network, is presented in detail in the accompanying paper. ${ }^{26} \mathrm{As}$ with the previous calculations, 23,25 there are a number of variables required in the tube model calculation of the correlation functions. The variable set used previously was:

1. The fraction of each chain which is contained in the $\mathrm{H}$-polymer crossbar and the fraction of arms which are labeled. These two variables are fixed by the reaction chemistry. For the present sample, the arms are fully "labeled" (with hydrogen-the crossbar is deuterium-labeled), and the crossbar constitutes a fraction $\mathrm{f}_{\text {cross }}=0.364$ of the whole polymer.

2. The monomer step length for Gaussian chain statistics. This can be measured in terms of a radius of gyration parameter by fitting the standard RPA result to a scattering experiment on an unstretched sample.

3. This calculation uses the Warner-E dwards model ${ }^{31}$ to describe the tube. In this model, each monomer is localized in space by a quadratic confining potential. The tube diameter, $\mathrm{d}_{0}$, used in this model, can in principle be obtained by fitting to the linear rheological response of the polymers. However, the precise correlation between the quadratic confining potentials (which constrain monomers in all three dimensions) and the melt tube (in which monomers can fluctuate along the tube length but are constrained in the two perpendicular directions) is unclear. It is doubtful whether the WarnerEdwards "tube diameter" and the melt tube diameter inferred from rheol ogy are the same. Neverthel ess, one would anticipate that they be of the same order of magnitude. Furthermore, the tube diameter should be scaled to account for tube dilation by constraint release as

$$
\mathrm{d}_{0}=\mathrm{d}_{00} \phi^{-\alpha / 2}
$$

where $d_{00}$ is the "undiluted" tube diameter and $\phi$ is the fraction of unrelaxed chain material at a given relaxation time. The exponent $\alpha$ is taken to be $4 / 3 .{ }^{22,32,33}$

4. The fraction of the arms that have relaxed (i.e., escaped the oriented tube) at a given relaxation time. An estimate of this can be obtained from the fitting the linear rheology spectrum using the theory of ref 22 , as is done above, giving the data of Figure 2. We should note that this relaxation spectrum is not necessarily appropriate for relaxations in the nonlinear regime. ${ }^{15}$

5. The degree of retraction of the arms. Following a nonlinear stretch, it is conjectured ${ }^{20}$ that the $\mathrm{H}$-polymer arms (which are stretched to above their equilibrium length in the tube by the Doi-Edwards factor ${ }^{1} \alpha(\mathbf{E})$ ) should retract back to their equilibrium length in the tube. There is no impediment to this motion, and the theoretically predicted time scale for this is the Rouse relaxation time of the arms. On the basis of the value of $\tau_{\mathrm{e}}$ used in fitting to linear rheology above, we estimate this time scale to be on the order of $5 \times 10^{-4} \mathrm{~s}$ for the present sample at ambient temperature. This corre sponds to about $4 \mathrm{~s}$ at $-40{ }^{\circ} \mathrm{C}$; i.e., the experimental stretching time is comparable to the stretch relaxation time of the arms. We do not expect, then, to be able to stretch fully the H-polymer arms in this experiment.

6. The degree of branchpoint withdrawal. The $\mathrm{H}$ polymer crossbar is, like the arms, stretched to above its equilibrium length in the tube. However, its retraction is impeded by the entropic penalty of pulling the branch points into the central tube. I deally, there would be no branchpoint withdrawal at stretches less than $\lambda$ $\approx 4$ (or, more precisely, tube el ongations less than $\alpha(\mathbf{E})$ $=2),{ }^{20}$ but there may be some branchpoint withdrawal due to local displacement of the branch point within the smooth elastic potential minimum of the tube branchpoint site. ${ }^{19}$ Such branchpoint withdrawal must be on the order of magnitude of a tube diameter (note that the tube diameter can increase with time due to tube dilation). The theoretically predicted time scale for branchpoint withdrawal is about the Rouse time of the whole polymer, which we estimate to be of the order of $4 \times 10^{-3} \mathrm{~s}$ at $298 \mathrm{~K}$. This is similar to the relaxation times probed in this experiment, so we would expect to see a transition from a state in which the branch points are not withdrawn to a state in which they are partially withdrawn.

In addition to the previous parameter set, in this present calculation we use extra parameters to represent the following physics. The first two of these could (and, indeed, should) have been included in previous calculations for the $\mathrm{H}$-polymer melt. ${ }^{22,23,25}$ The third is the only extra parameter required in the new theory. A fourth parameter, related to polydispersity in the sample, is discussed in the subsection bel ow.

7. In this experiment there is independent evidence (from SEC and TGIC) of a significant quantity of two major impurities: an " $\mathrm{H}$ "-polymer with an arm missing (i.e., a star polymer with one long arm) and an " $\mathrm{H}$ "polymer with a double-backbone unit. These are present in sufficiently large quantities to affect the scattering and so must be included in the calculation. This involves calculating correlation functions for these impurities and adding these to the correlation function of the "pure" H-polymer in an appropriately weighted manner. One must also include parameters for the degree of retraction of the long star arm (this relaxes on a similar time scale to the whole $\mathrm{H}$-polymer), the fraction of this arm that has relaxed orientationally (since this arm, combining the $\mathrm{H}$-polymer backbone and an $\mathrm{H}$-polymer arm, is about 3 times longer than the $\mathrm{H}$-polymer arms, we expect the relaxed fraction of this arm to be smaller than for the $\mathrm{H}$-arms), and the degree of branchpoint withdrawal of the double-backbone $\mathrm{H}$-polymer. The fraction $\phi$ of unrelaxed material used in eq 1 for the tube 
dilation takes into account relaxation of the impurities. The impurities were not included in the fit to linear rheological data of Figure 1, and so we cannot make exact predicitions for the values of these parameters. However, the overall scattering does not appear to be very sensitive to the choice of these parameters, solong as they are given reasonable values. The main effect of the impurities is to increase the amount of scattering at small wavevectors below the peak.

8. It has been predicted, in the case of polymer networks, that the magnitude of the localizing potentials in the Warner-Edwards model should deform affinely with the strain, ${ }^{34-37}$ such that the Warner-Edwards "tube diameter" in the Cartesian direction $\mu$ varies as

$$
\mathrm{d}_{\mu}=\mathrm{d}_{0} \lambda_{\mu}^{v}
$$

where $\lambda_{\mu}$ is the macroscopic stretch ratio in this Cartesian direction and the exponent $v$ is predicted to take a value of $1 / 2$ in the case of networks. It should be noted that the Warner-Edwards model is a somewhat artificial construct, especially in the case of melts as indicated above. Bearing this in mind, we have allowed ourselves a choice of the exponent $v$; typically we have tried both $v=0$ and $v=1 / 2$.

9. The new theory ${ }^{26}$ requires just one additional parameter, $\mathrm{N} / \mathrm{N}_{\mathrm{e}}$, which is the ratio of a reference degree of polymerization, $\mathrm{N}$, to the entanglement molecular weight, $\mathrm{N}_{\mathrm{e}}$, giving $\mathrm{N} / \mathrm{N}_{\mathrm{e}}=78$ for $\mathrm{M}_{\mathrm{e}}=2000 \mathrm{~g} \mathrm{~mol}^{-1}$. The typical degree of polymerization of the polymers $(\mathrm{N})$ determines the magnitude of composition fluctuations in the system. As shown by a scaling argument in the Appendix, the ratio $\mathrm{N} / \mathrm{N}_{\mathrm{e}}$ sets the ratio between composition and elastic fluctuations in the system. Although $\mathrm{Ne}_{\mathrm{e}}$ is related to the tube diameter, we noted above that the Warner-Edwards tube diameter is a poorly understood quantity, so we keep the variables $\mathrm{N}_{\mathrm{e}}$ and $\mathrm{d}_{0}$ as separate variables. (Nevertheless, we expect $\mathrm{d}_{0}{ }^{2} \approx \mathrm{N}_{\mathrm{e}} \mathrm{b}^{2}$ at least in order of magnitude.) J ust as the tube diameter is dilated by constraint release via eq 1 , so $\mathrm{N}_{\mathrm{e}}$ must vary as

$$
\mathrm{N}_{\mathrm{e}}=\mathrm{N}_{\mathrm{e} 0} \phi^{-\alpha}
$$

It is clear from the above that, while there are many variables in the model, all of them are either fixed or remain strongly constrained by physical considerations respecting the tube model and the results of rheological experiments and SANS on the undeformed sample.

Polydispersity Correction. The experimental data (Figures 4 and 6) showed a significant amount of scattering in the low wavevector region, below the scattering peak, that could not wholly be accounted for simply by the inclusion of impurities (i.e., "polydispersity" in composition) at the levels detailed above. Since a higher impurity level would not be consistent with the SE C and TGIC characterization of the sample, one must consider alternative sources of low-angle scattering. One such source is polydispersity of individual block lengths in the sample. One would expect each of the constituent "blocks" (i.e., each of the arms and the backbone) in the sample to be independently polydisperse, and it is wellknown that even a small amount of such polydispersity in block copolymer samples can produce a significant degree of low-angle scattering. This occurs because variation in the molecular weight of each of the blocks produces polymers containing slightly differing fractions

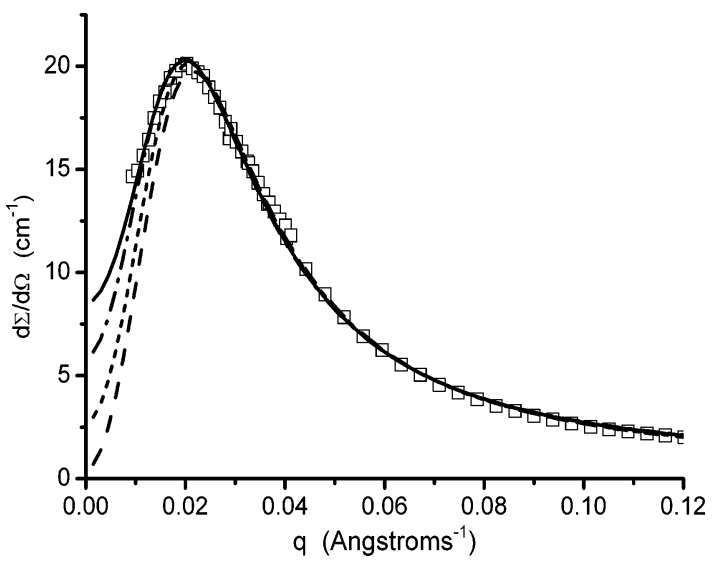

Figure 4. Radially averaged scattering intensity from the isotropic sample, together with theoretical fits using the standard RPA theory, monodisperse without impurities (long dashes), monodisperse with impurities (short dashes), with polydispersity correction using $\epsilon=0.38$ (dash-dot), and with approximate polydispersity correction using $a=0.2$ (solid line).
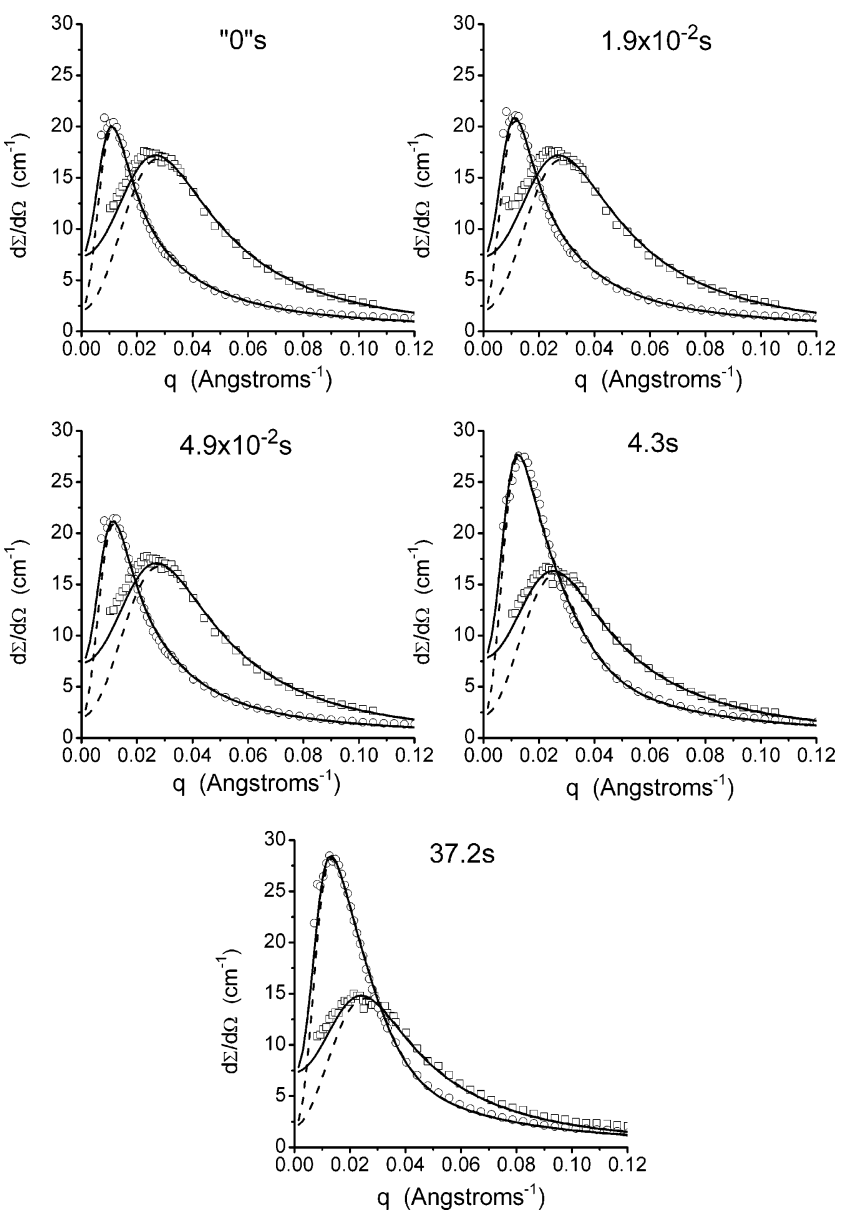

Figure 5. Scattering data parallel (circles) and perpendicular (squares) to the stretch at each relaxation time, together with theoretical fits to the data with (solid lines) and without (dashed lines) the approximate polydispersity correction, using $a=0.2$. Other fitting parameters are as given in Table 1.

of labeled monomers. The random distribution of such polymers through the sample volume produces small variations in composition at large length scales, giving rise to low-angle scattering.

It is quite straightforward to include polydispersity of the blocks in the standard RPA theory used to calculate the scattering from the unstretched melt. (This type of calculation was first performed by Leibler and 


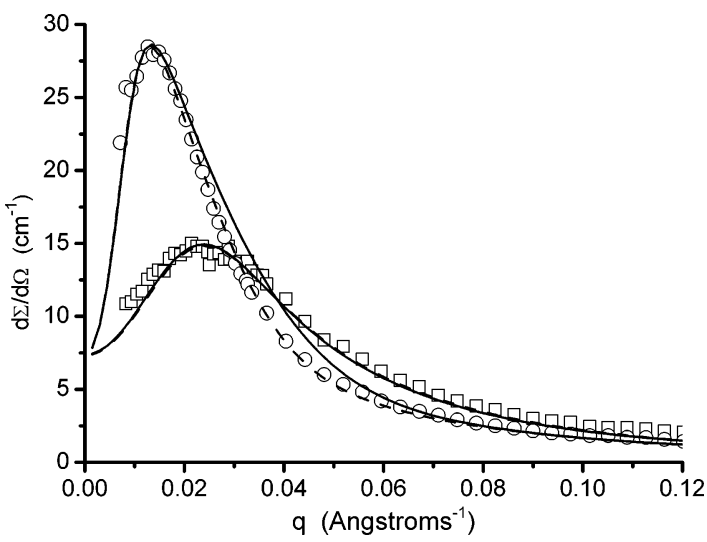

Figure 6. Comparison between fits with $v=0$ (solid line) and $v=1 / 2$ (dashed line) for the data at an annealing time of 37.2 $\mathrm{s}$. The fit for $v=0$ uses $\mathrm{x}=0.84$ and $\mathrm{X}_{\mathrm{L}}=0.4$ but otherwise uses the same parameters as given for the $v=1 / 2$ fit in Table 1.

Benoit. ${ }^{38}$ ) For the sake of simplicity we assume that each of the constituent blocks has a degree of polymerization, distributed uniformly between $\mathrm{N}_{\mathrm{bo}}(1-\epsilon)$ and $\mathrm{N}_{\mathrm{bo}}(1+\epsilon)$. The length of each block is assumed to vary independently of the others. Under this assumption, all constituent blocks have the same polydispersity, which is

$$
\left.\frac{\mathrm{M}_{\mathrm{w}}}{\mathrm{M}_{\mathrm{n}}}\right|_{\text {block }}=1+\frac{\epsilon^{2}}{3}
$$

The polydispersity of the whole H-polymer is somewhat smaller:

$$
\left.\frac{M_{w}}{M_{n}}\right|_{H-\text { polymer }}=1+\frac{\epsilon^{2}}{12}\left(1+5 f_{\text {cross }}^{2}-2 f_{\text {cross }}\right)
$$

The results of such an RPA calculation are presented bel ow for the unstretched sample. Note that quite small polydispersities are obtained from quite large values of $\epsilon$ (e.g., block polydispersities of 1.05 and $\mathrm{H}$-polymer polydispersities of 1.01 are obtained from $\epsilon=0.4$ ).

The inclusion of polydispersity as described above (i.e., each block independently polydisperse) in the full RPA theory used to model the stretched sample would constitute a lengthy addition to an al ready complicated theory. For this reason, we seek a method that will approximately correct for small levels of polydispersity in the sample but that will not unduly lengthen the coding and execution of the numerical calculation. One way to mimic the effect of polydispersity is to "artificially" vary the fraction of label ed monomers on each of the polymers. We do this by modeling the melt as a blend of three separate populations of chains: (i) in which all the chains have the degree of polymerization and architecture defined by the mean parameters of the melt; (ii) in which all the labeled blocks are increased in length by a factor $(1+$ a) but the unlabeled ones are decreased in length by a factor ( $1-a)$; and (iii) in which all the unlabeled blocks are increased in length by a factor $(1+a)$ but the labeled ones are decreased in length by a factor $(1-a)$. These three populations are included with equal number fraction in the calculation of correlation functions for the sample. Each population contains both the $\mathrm{H}$-polymer and impurities at the levels listed in part 7 above. By restricting the number of such chain populations to just three, we are avoiding the additional multidimensional integral over the independent distributions of block lengths that would be needed in each of the correlation functions to account for polydispersity correctly! The small parameter a is chosen, as detailed below, so that the predicted scattering for the unstretched melt approximately matches the result of the standard RPA theory with the more realistic polydispersity calculation-in practice, both are fitted to the scattering data on the unstretched sample. As will be shown below, the value of a obtained through this procedure is of the same order as, but a little smaller than, the value of $\epsilon$. Since this is an approximate way of including polydispersity effects, we shall present theoretical results both with and without this correction, so that the reader can obtain an indication of the magnitude of the effect.

\section{Results and Comparison with Theory}

4.1. The Unstretched Sample. Figure 4 shows the scattering data for the unstretched sample, together with theoretical fits to the data, using the standard RPA theory. Four fits are shown. The first simply uses the scattering prediction for a pure H-polymer sample with monodisperse blocks. Evidently the fit is reasonable in the peak and the high-q region, but the fit is not good in the low-q region. The second fit includes $25 \%$ vol ume fraction of the three-arm impurity and 5\% volume fraction of the double-crossbar impurity. These quantities are the maximum allowable from SEC and TGIC data on the sample. While this improves the fit a little to the left of the peak, there is still not enough scattering predicted in this region. The third fit uses the same quantities of impurities but allows each block to vary independently in length as described above, with a parameter $\epsilon=0.38$ (giving block polydispersities of 1.05 and an $\mathrm{H}$-polymer polydispersity of 1.01-these values appear to be quite consistent with SEC analysis of the sample). Note that even this small amount of polydispersity can have quite a dramatic effect on the low-angle scattering, so that the data are now quite well modeled. It would appear that a reasonable description of this sample, from the point of view of a scattering calculation, is to include the impurities at this level and also to all ow for polydispersity in the blocks.

The fourth fit shown in the figure uses the approximate polydispersity correction described above, blending three populations of chains each with a slightly different fraction of labeled polymer. The polydispersity parameter in this fit takes a value of $a=0.2$. Evidently, this gives quite a similar scattering prediction to that obtained from the more correct theory, especially in the wavevector range of the data. (The largest discrepancy is at very low wavevectors, where there is no corresponding data.) We shall use this approximate method for accounting for polydispersity, with the same value of $a=0.2$, to model the scattering data from the stretched sample below. We suggest that the results obtained from this approximate method are similar to what would be obtained from a more accurate representation of the polydispersity.

The four fits shown to the undeformed data all use the same value for radius of gyration as a function of molecular weight. The radius of gyration of the $\mathrm{H}$ polymer crossbar in this sample is determined to be 90 $\AA$, which yields for linear polybutadiene polymers

$$
R_{g}=0.376 \sqrt{M}
$$


and $\mathrm{C}_{\infty}=7 \pm 1$. This is in good agreement with the scaling law and the value of the characteristic ratio given in the literature for linear polybutadiene polymers of similar $1,2-1,4$ microstructure. ${ }^{29}$ Hence, the radius of gyration of a linear polymer with the same mol ecular weight (157.2 kg mol$\left.{ }^{-1}\right)$ as the $\mathrm{H}$-polymer used here would be $145 \AA$. (This is the $\mathrm{R}_{\mathrm{g}}$ used in the definition of the parameter $s$ in the accompanying theoretical paper. ${ }^{26}$ )

4.2. Sample Stretched by a F actor $\lambda=2$. Figure 5 shows scattering data both parallel and perpendicular to a uniaxial stretch by a factor $\lambda=2$, both immediately after the stretch and at various relaxation times following the stretch. The relaxation time range is supposed to cover almost the complete arm relaxation domain, as predicted by fitting to linear rheology in oscillatory shear (see Figure 2). Also shown are theoretical fits to the scattering data, using the new theory, both with and without the approximate polydispersity correction. It can be seen that the evolution of the scattering is qualitatively quite similar to that seen in the previous tip-labeled polyisoprene sample. ${ }^{23}$ The scattering immediately following the stretch is almost an affine transformation of the undeformed scattering. The peak parallel to the stretch is moved toward the origin, roughly by a factor of 2 as compared to the peak in the isotropic scattering. The peak perpendicular to the stretch has moved away from the origin, but by less than the factor of $\sqrt{2}$ that would be commensurate with a purely affine transformation from the isotropic state. Additionally, the perpendicular peak is of slightly lower magnitude than the parallel one. These two factors indicate that a certain degree of relaxation has al ready occurred at the smallest length scales, which reduces the perpendicular scattering at higher wavevectors. In common with the polyisoprene sample at a $\lambda=$ 3 , the peak scattering at the longest relaxation times exhibits a sharp increase in the scattering parallel to the stretch together with a mild decrease perpendicular to the stretch.

One feature of the scattering from this sample, which was not present in the previous polyisoprene data, is that the scattering does not appear to change a great deal in the early stages of relaxation. In the first three data sets, there is some tendency for the high wavevector scattering to relax toward isotropy, but this does not occur to the same extent as occurred in the "tip-labeled" polyisoprene sample. In the late-time regime, the former PI sample was al most isotropic in the high-q region, but this is not the case here. These differences can be understood in terms of the different labeling of the two samples. In the present sample, one of the components (the deuterium-labeled polymer in the crossbar) is wholly confined to the tube for the relaxation times probed here. Hence, this component remains strongly anisotropic and is held in this configuration by the tube constraints. The relaxed portions of chain contain only hydrogen-label ed monomers from the arms; these portions of the polymer are constrained, by excludedvolume interactions, to "fill in the gaps" between the anisotropic, deuterium-labeled crossbar monomershence, the overall scattering remains quite anisotropic even in the large wavevector region. Moreover, since the scattering is dominated by the constrained crossbars, it does not change a great deal until there is sufficient relaxation for the increase in low-q parallel scattering due to (we suppose) elastic fluctuations and inhomogeneities of the entanglement network.

In the former tip-labeled PI sample, however, once the arm relaxation had proceeded beyond the small labeled tips, the orientationally relaxed sections of chain contained both labeled and unlabeled species. Although these relaxed portions of chain are still forced by excluded volume to "fill in the gaps" between the anisotropic, tube-constrained sections of chain, the fact that they contain both labeled and unlabeled species means the resultant effect on the scattering is less strong, and the high wavevector scattering becomes increasingly isotropic.

Since the crossbar dominates the scattering for the three earliest times and the arms are not sufficiently relaxed for elastic inhomogeneities to have a large effect, it is possible to obtain a good fit to these data using either the earlier RPA theory ${ }^{25}$ or the (much simpler) "factorizable RPA" theory ${ }^{30}$ used to model the polyisoprene data. ${ }^{23}$ These earlier theories, however, cannot fit the full set of relaxation data for this sample; specifically, they do not predict the strong increase in peak anisotropy at late times. We know of no earlier theory that is able to provide a reasonable and consistent fit to the full set of data.

The new theory, including the effects of elastic inhomogeneities in the entanglement network, provides an excellent fit to the along-axis data of Figure 5. In particular, it reproduces the large increase in the peak anisotropy at late times. While the fits shown are quantitatively very good, the qualitative feature of increasing peak anisotropy is reproduced for a wide range of parameter choices. This gives one a good deal of confidence in interpreting the physics underlying this prediction, and in the central conclusion of this paper that the elastic inhomogeneities dominate the small wavevector scattering at late times, by giving rise to this increasing peak anisotropy. The physical mechanism at work is very similar to that which gives the "butterfly" patterns in stretched networks containing solvent molecules. There are frozen-in stresses due to fluctuations in both local tube concentration and orientation, which favor local contractions of the network in some regions and (by continuity) local expansions of the network in other places. In a system that is nearly incompressible overall, these network contractions and expansions can only occur if there is some mobile species which can leave the contracting regions and flow into the expanding regions. In the case of swollen networks, the solvent is the mobile species. In the particular case where the "solvent" is a free polymer chain, one must wait until times greater than the relaxation time of the polymer chain before the "butterfly" patterns appear; 13 at shorter times the chains are not sufficiently mobile to allow the network motion. Similarly, in the present sample one must wait until the $\mathrm{H}$-polymer arms are sufficiently relaxed that they can move from contracting regions of the entanglement network toward expanding regions. However, even once they are relaxed to this degree, the $\mathrm{H}$-polymer arms are not able to move large distances because they are chemically joined to sections of chain that are still confined to the tube. For this reason, the "butterfly" patterns can only develop at finite wavevectors, commensurate with the radius of gyration of the relaxed polymer arms, and at these wavevectors one observes an enhanced peak in the scattering. 
Table 1. Fitting Parameters at a Sample Deformation of $\lambda=2$

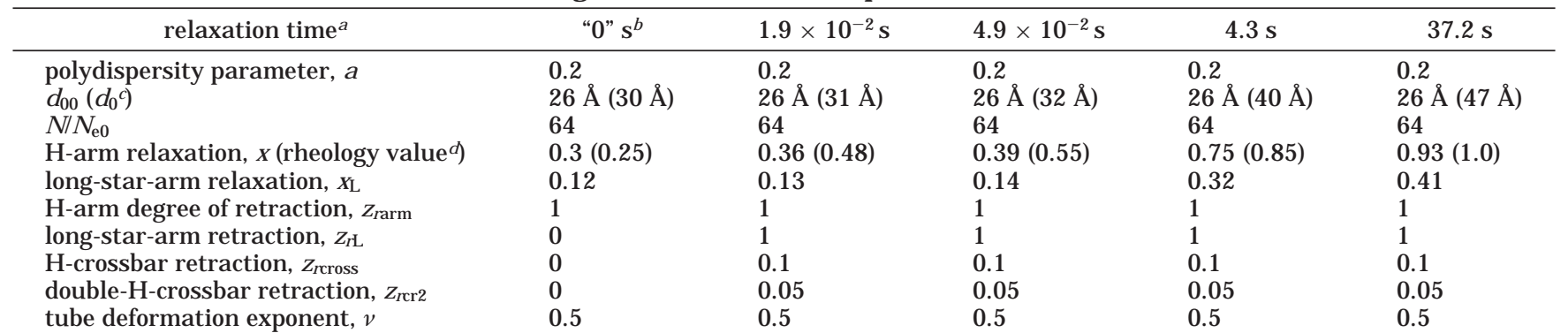

a Relaxation time at room temperature based on WLF shifting of time and temperature data. ${ }^{b}$ Estimated relaxation time is $10^{-3} \mathrm{~s}$. c Tube diameter after dilution. d Rheology values based on fits to oscillatory linear rheology data.

It is clear that the approximate inclusion of polydispersity, in the manner described above, gives a large improvement to the fit, especially perpendicular to the stretch. In fact, the fit could be improved further by the inclusion of a slightly higher degree of polydispersity, but we decided to fix the value at $a=0.2$ for consistency with the scattering data from the unstretched sample. Polydispersity has a particularly large effect on the perpendicular peak for the following reason. A purely affine transformation of the sample upon stretching would result in a perpendicular peak that was at a larger wavevector, and considerably higher intensity, than the peak seen here. However, fluctuations of the polymers within the tube reduce the composition variations at these large wavevectors, so that only the lefthand side of the affinely transformed peak remains. It is in this region (of the affinely transformed peak) that polydispersity has its largest effect, resulting in an increase in the low-angle scattering. There is a corresponding increase in the peak scattering perpendicular to the stretch in the deformed sample because this "peak" arises from the same composition variations that contributed to the low-angle side of the peak in the isotropic scattering. We note that this is a direct result of the fact that the tube diameter and polymer radius of gyration are of a similar order of magnitude. If the tube diameter were relatively much smaller, we would expect to see a perpendicular peak that was larger in magnitude, at higher wavevectors, and much less affected by polydispersity.

The set of parameters used to obtain the fits in Figure 5 are given in Table 1 . While there are numerous parameters reported here, these are required to represent the tube model picture of relaxation in this melt and consequently should not be treated as free fitting parameters. As stated above, the polydispersity parameter was fixed at the value obtained from the unstretched melt. The value of $\mathrm{N} / \mathrm{N}_{\mathrm{e} 0}=64$ was initially fixed at this reasonable value and not subsequently adjusted for any of the data fits. (I n practice, we suspect that good fits could also be obtained if this parameter were allowed to vary, say by $20-30 \%$.) As described above, the polydispersity parameter is fixed by the data from the unstretched sample. The undiluted "tube diameter" $\mathrm{d}_{00}$ and tube deformation exponent $v$ were allowed to vary within a reasonable range to achieve the fits, but we required that the same value be used for the full set of data, as is clear from Table 1 . As discussed in more detail below, the retraction parameters were set at sensible values based on the expected relaxation times for the processes they represent.

The remaining parameters exhibit a good consistency with those obtained through linear rheology. The frac- tion, $x$, of the arm relaxed at each time is consistently within 0.16 of the value obtained through linear rheology, and a comparison between the two is shown in Figure 2. This appears reasonable, given the approximations that are used in the scattering calculation. (In particular, the Warner-Edwards model is not a true depiction of the melt tube, and one would really like to include physics such as fluctuation of the monomers along the tube contour and the fact that there should be a distribution of relaxed fractions, $x$, at each relaxation time.) We consider that the largest error in these reported values for $x$ arises from these inherent approximations in the model. It is reasonable to assign an "error bound" in the theoretical value of x commensurate with one entanglement molecular weight al ong the tube (i.e., an error of $\Delta x \approx 0.1$ for the undiluted tube and larger error bounds after tube dilation). Moreover, there is an error of at least $\Delta x=0.1$ in the corresponding value of $x$ reported from linear rheology. (Errors arise both in the theor etical fitting to the linear rheology of Figure 1 and in the WLF shifting used to convert the sample's complex temperature-time history into an equivalent relaxation time at room temperature.) There is an apparent systematic error in Figure 2. (The SANS $x$ value is, apart from the first data point, consistently smaller than the rheology value.) Such systematic differences could be caused by approximations in the theory or by the sample temperature being a typically little lower than that of the surroundings during the heat/cool cycle. In any case, the difference between the linear rheol ogy $x$ value and the value used in the SANS fitting is consistent with the error bounds suggested above.

No specific rheology data were available for the longstar-arm relaxation parameter, $\mathrm{X}_{\mathrm{L}}$, but this is constrained by the tube model to be less than $\mathrm{x}$ (longer arms relax more slowly than short ones) but greater than $x\left[f_{\text {arm }} /\left(f_{\text {cross }}+f_{\text {arm }}\right)\right]$ because a longer arm gives a weaker potential for the activated relaxation of branched polymer arms. In practice, $x_{L}$ was set at a sensible intermediate value between these limits, and since it is a single arm on a minority component, the predicted scattering is not sensitive to the precise value used.

The amounts of retraction of the arms and backbone were not treated as free parameters, but fixed at values commensurate with the expected relaxation times for these processes. In Table 1, these retractions are reported as a "degree of retraction", $z_{r}$, defined so that the chains are retracted within the tube by a factor

$$
\gamma=z_{r}(\alpha(E)-1)+1
$$

where $\alpha(\mathbf{E})$ is the Doi-E dwards tube el ongation factor. 

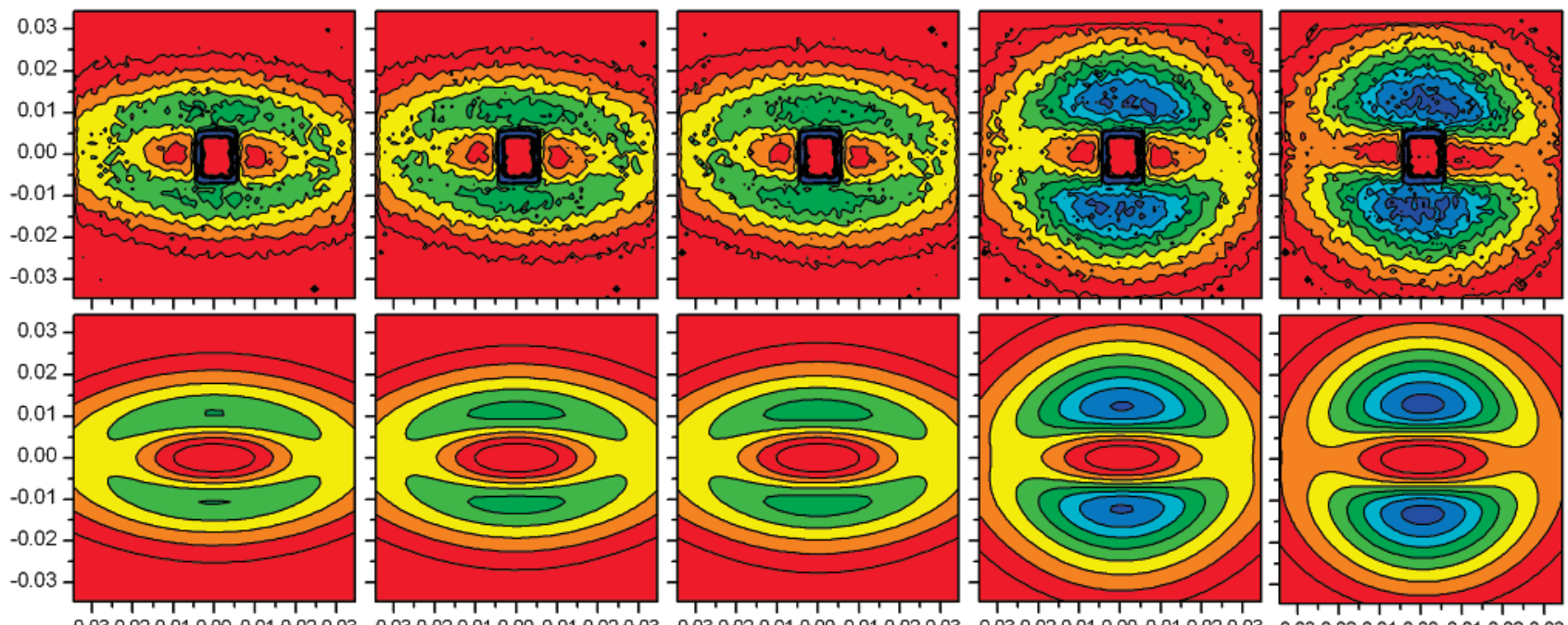

Figure 7. Top: 2D scattering for the sample after a stretch of $\lambda=2$ with increasing relaxation time from left to right. B ottom: theoretically predicted 2D scattering, using the same parameters as gave the fits of Figure 5 (in Table 1 ). Contours are evenly spaced at intensities of $10,12.5,15, \ldots ., 27.5 \mathrm{~cm}^{-1}$. The stretch direction is vertical.

Hence, a value of $z_{r}=0$ represents an unretracted chain, whereas a value of $\mathrm{Zr}_{\mathrm{r}}=1$ represents a chain that is "fully" retracted by the Doi-E dwards factor, $\alpha(\mathbf{E})$. The arms were treated either as unretracted $\left(z_{r}=0\right)$ or fully retracted $\left(z_{r}=1\right)$, and in fact only the retraction time of the long-star-arm impurity falls within the relaxation time range of the experiment. The values of $z_{r}$ used at later times for the crossbar retraction are representative of a retraction on the order of a single tube diameter. Hence, the scattering patterns here are consistent with the idea of a partial branchpoint withdrawal. However, it appears that the scattering is more sensitive to the elastic inhomogeneities than to this branchpoint withdrawal-it would be possible to obtain a reasonable fit to the data by ignoring the branchpoint withdrawal and slightly increasing the value of $x$.

Of particular note is the fact that the fits shown use a deformed Warner-Edwards tube diameter, with exponent $v=1 / 2$ (this value is as predicted for polymer networks $\left.{ }^{34-37}\right)$. Although the i sotropic tube diameter ( $v$ $=0)$ gives a good qualitative description of the data (including the increasing peak anisotropy), the quantitative fit is not quite so good, especially at the late times. In particular, as shown in Figure 6, the shape of the parallel peak for $v=0$ is not quite reproduced, and the high wavevector scattering is a bit too isotropic. It is reasonable to say that, within the approximations of the present model, the data overall are more consistent with the deformed tube diameter, $v=1 / 2$. However, it must be stressed that there are a number of approximations made in the calculation of the various correlation functions in the theory (more detail is given in the accompanying paper), to the extent that one must be quite cautious in drawing firm conclusions about such parameters as $v$. It is more important that the broad qualitative features of the scattering development are predicted well by the theory, as indeed they are. Nevertheless, we note that independent measurements by coherent NSE experiments on partially labeled deformed polymers ${ }^{39}$ agree with the nonaffine mechanism without relying on the Warner-E dwards model.

Figure 7 shows the full 2D scattering intensity for the $7 \mathrm{~m}$ detector distance at each of the measured times. Shown alongside is the predicted 2D scattering using the theory with the polydispersity correction. These plots are generated by taking the fitting parameters from the along-axis plots, given in Table 1, and extending the prediction in the 2D plane. Given that the majority of these 2D data were not used in the alongaxis fits, the reproduction of the contours in both shape and intensity is remarkable. The fit, especial ly in terms of the shape of the parallel peak, remains better for $v$ $=1 / 2$ than for $v=0$ (not shown), al though the qualitative features are reproduced by either choice of parameter.

\section{Conclusions}

We have presented scattering data from a melt of $\mathrm{H}$-shaped polybutadiene polymers in which the central crossbar was wholly labeled with deuterium. The data monitor the relaxation of this melt following a uniaxial step strain by a factor $\lambda=2$. By comparison with a new theory, presented in the accompanying paper, ${ }^{26}$ we have shown that these data are wholly consistent with the relaxation mechanisms predicted by the tube model, provided one includes the additional contributions due to elastic fluctuations and inhomogeneities in the entanglement network. These are the same inhomoge neities that give rise to the "butterfly" scattering in stretched networks and asymmetric melts. The new mechanism suggested in the present model is that these couple to the motion of the relaxed arms in the branched polymer melt, giving an enhanced scattering parallel to the stretch, at wavevectors commensurate with the radius of gyration of these arms, and which increases with increasing arm relaxation. Although this qualititative feature is predicted for a wide range of choices of parameter in the model, it appears that the best fits to the data can be obtained when the Warner-Edwards tube diameter used in the scattering calculation is deformed by the strain, with an exponent $v=1 / 2$ identical to that predicted for polymer networks. ${ }^{34-37}$ It is possible, however, that this is a feature of the approximations made in the calculation, and this particular result should be treated with appropriate caution (especially since we have probed only one value of the strain in these data!).

It is clear that while this experiment and similar previous ones have revealed some interesting physical phenomena, they are not so "clean" in the detection of 
the tube model phenomena for which they were originally proposed ${ }^{24-i t}$ is the elastic inhomogeneities rather than branchpoint withdrawal that appears to dominate the scattering. For this reason, it seems unlikely that experiments such as this one would be able to distinguish between subtle differences in rheology models (e.g., tube dilation vs more refined models of constraint release ${ }^{40,41}$ or different modes of branchpoint withdrawal).

It is to be presumed, then, that such elastic inhomogeneities are present and active in all long-chain branched polymer melts and that the motion of the more rapidly relaxing portions of these chains is influenced by them. Certainly, as demonstrated here, they are important for, and must be included in, scattering calculations on such melts. Moreover, these experiments raise the important question as to whether the elastic inhomogeneities are important as regards the rheological properties of long-chain branched polymer melts. It is certain that they must have some effect; they are present in the melt free energy functional, and their behavior depends on the melt deformation. However, we would argue that their overall effect is likely to be small for the following reason. In this scattering experiment, we are measuring relatively small fluctuations about the average composition of the melt-the scattering experiment does not directly measure this "average" composition. Rheological experiments, however, are "zero-wavevector" experiments and measure, primarily, the average state of the melt. As far as the stress is concerned, the average state is the stretched state predicted by the tube model, and the elastic inhomoge neities give rise to small fluctuations about this state, constituting a small correction to the average rheol ogical behavior. Having said this, it is clear that the inhomogeneities do couple to the motion of the relaxing arms, and it is possible that they might affect, for example, the relaxation processes, and especially the relaxation times, of these arms. This, then, must be the subject of future investigation.

While the fit of the theory to these present data is extremely good, there remain discrepancies between the theory and the previously published polyisoprene data ${ }^{23}$ at the longest times of relaxation, at larger stretches $(\lambda$ $=3$ ) than performed here. These discrepancies are described in more detail in the accompanying paper. ${ }^{26}$ It is speculated that there are physical mechanisms not included in the present model that become more apparent at larger stretches. This clearly points to the need for a more extensive study (covering a variety of stretches, chemistries and labeling, and including mechanical analysis and elongational viscosity measurements) than we have been able to achieve and present to date.

Acknowledgment. The experiments reported here were performed with financial support in the framework of The BRITE EURAM Project BR-97-4248, "LCB Polyol efins". We thank St. Kahle (J ülich) for the dielectric measurements. D. J. Read and T. C. B. McLeish gratefully acknowledge the financial support of an EPSRC Advanced Research Fellowship and Senior Research Fellowship, respectively.

\section{Appendix A. Scaling Argument for the Effect of Elastic Inhomogeneities}

In this Appendix we present a scaling argument to demonstrate how the parameter $\mathrm{N} / \mathrm{N}_{\mathrm{e}}$ arises when considering the effect of elastic inhomogeneities on monomer density fluctuations in a scattering calculation. We consider a polymeric system in which the typical degree of polymerization is $\mathrm{N}$ and where the typical number of monomers per unit volume is $\rho$. We consider the typical fluctuations $\Delta \mathrm{n}$ in the number, $\mathrm{n}$, of monomers present in a reference volume V. (Since we are presenting a scaling argument only, we shall be ambiguous here as to the particular labeling of the polymers and whether we are counting label ed monomers only-the scaling results below will be modified in any more detailed calculation by prefactors which depend on the polymer structure and volume fractions of label ed species.)

Scattering intensities are proportional to the meansquare fluctuation $\left\langle\Delta n^{2}\right\rangle$. For ordinary composition fluctuations, $\left\langle\Delta n^{2}\right\rangle$ is proportional to the typical number of polymers $\mathrm{V} \rho / \mathrm{N}$ present in the volume $\mathrm{V}$ (which gives the fluctuations in number of polymers in the reference volume $\mathrm{V}$ ) multiplied by $\mathrm{N}^{2}$ (to convert from number of polymers to number of monomers):

$$
\left\langle\Delta \mathrm{n}^{2}\right\rangle_{1} \sim \mathrm{V} \rho \mathrm{N}
$$

To assess the effect of elastic inhomogeneities for a material with entanglement degree of polymerization $\mathrm{N}_{\mathrm{e}}$, we balance the typical modulus of the material

$$
\mathrm{G} \sim \frac{\rho \mathrm{k}_{\mathrm{B}} \mathrm{T}}{\mathrm{N}_{\mathrm{e}}}
$$

with estimates for the stress fluctuations in the system.

The first contribution to stress fluctuations we consider is fluctuations in the random orientation of tube segments. E ach tube segment is independently oriented, so that there are random fluctuations in stress arising from tube segments in one region being more oriented than those in another. The contribution to the average stress over a volume $\mathrm{V}$ from a single tube segment within that volume is proportional to $\mathrm{k}_{\mathrm{B}} \mathrm{T} N$. The second moment of stress fluctuations $\left\langle\Delta \sigma^{2}\right\rangle$ due to random orientation of tube segments in that volume is proportional to the typical number of tube segments in that volume, $\mathrm{V} \rho / \mathrm{N}_{\mathrm{e}}$, and to the square of the typical stress contribution from a single segment $\left(k_{B} T / N\right)^{2}$. Hence

$$
\left\langle\Delta \sigma^{2}\right\rangle \sim\left(\frac{\mathrm{k}_{\mathrm{B}} T}{\mathrm{~V}}\right)^{2} \frac{\mathrm{V} \rho}{\mathrm{N}_{\mathrm{e}}} \sim \frac{\left(\mathrm{k}_{\mathrm{B}} T\right)^{2} \rho}{\mathrm{VN}_{\mathrm{e}}}
$$

which gives rise to fluctuations in network strain $\left\langle\Delta \epsilon^{2}\right\rangle$ of order

$$
\left\langle\Delta \epsilon^{2}\right\rangle \sim \frac{\left\langle\Delta \sigma^{2}\right\rangle}{\mathrm{G}^{2}} \sim \frac{\mathrm{N}_{\mathrm{e}}}{\rho \mathrm{V}}
$$

and hence fluctuations in monomer number of order

$$
\left\langle\Delta \mathrm{n}^{2}\right\rangle_{2} \sim \mathrm{n}^{2}\left\langle\Delta \epsilon^{2}\right\rangle \sim \mathrm{V}_{\rho} \mathrm{N}_{\mathrm{e}}
$$

Comparing eqs 8 and 12 shows that the ratio of the effects of ordinary composition fluctuations to elastic fluctuations of the type discussed above is of order $\mathrm{N} / \mathrm{N}_{\mathrm{e}}$, and for this reason such a parameter is required in any theory of this kind.

The accompanying paper ${ }^{26}$ additionally considers a second source of stress fluctuations due to fluctuations 
in density of tubes. Since, after some relaxation of the polymers, the active tubes cover only part of the polymer chains (i.e., the crossbar and inner sections of the arms), there can be fluctuations in the density of tube segments, in the same way as there are fluctuations in the density of label ed monomers in a partially labeled block copolymer melt. The typical fluctuations $\left\langle\Delta \mathrm{n}_{\mathrm{seg}}{ }^{2}\right\rangle$ in the number, $\mathrm{n}_{\text {seg, }}$ of tube segments present in the reference volume $V$ due to this mechanism is proportional to the typical number of polymers $\mathrm{V} \rho / \mathrm{N}$ present in the volume $V$ (which gives the fluctuations in number of polymers in the volume) multiplied by $\left(\mathrm{N} / \mathrm{N}_{\mathrm{e}}\right)^{2}$ (to convert from number of polymers to number of tube segments)

$$
\left\langle\Delta \mathrm{n}_{\mathrm{seg}}{ }^{2}\right\rangle \sim \frac{\mathrm{V} \rho \mathrm{N}}{\mathrm{N}_{\mathrm{e}}^{2}}
$$

and since each segment contributes a stress proportional to $k_{B} T N$, the resultant fluctuations in stress are of order

$$
\left\langle\Delta \sigma^{2}\right\rangle \sim\left(\frac{\mathrm{k}_{\mathrm{B}} \mathrm{T}}{\mathrm{V}}\right)^{2}\left\langle\Delta \mathrm{n}_{\mathrm{seg}}{ }^{2}\right\rangle \sim \frac{\left(\mathrm{k}_{\mathrm{B}} \mathrm{T}\right)^{2} \rho \mathrm{N}}{\mathrm{VN}_{\mathrm{e}}{ }^{2}}
$$

Following the same line of reasoning as above, this leads to fluctuations in monomer number of order

$$
\left\langle\Delta \mathrm{n}^{2}\right\rangle_{3} \sim \mathrm{V} \rho \mathrm{N}
$$

i.e., this contribution scales in the same way as eq 8. It obtains an extra factor of $\mathrm{N} / \mathrm{N}_{\mathrm{e}}$ above eq 12 because the positions of tube segments are correlated over the whole polymer, which increases the fluctuations in tube segment density by this same factor. Having said this, the numerical prefactor to the fluctuations in eq 15 is typically quite small because (i) fluctuations in density of polymers, and hence of tube segments, are suppressed by excluded-volume interactions in the melt and (ii) in the detailed calculation the prefactor for these stress fluctuations is identically zero in the limit of no stretch and is small for small finite stretches. This mechanism is discussed in more detail in the accompanying paper. ${ }^{26}$

\section{References and Notes}

(1) Doi, M.; Edwards, S. F. The Theory of Polymer Dynamics; Clarendon: Oxford, 1986.

(2) Likhtman, A. E.; McLeish, T. C. B. Macromolecules 2002, 35, 6332.

(3) Doi, M. J . Polym. Sci., Polym. Lett. Ed. 1981, 19, 265.

(4) Doi, M. J . Polym. Sci., Polym. Phys. Ed. 1983, 21, 667.

(5) Marrucci, G. J. Non-Newtonian Fluid Mech. 2000, 95, 369

(6) Mead, D. W.; Larson, R. G.; Doi, M. Macromolecules 1998, 31, 7895.
(7) Milner, S. T.; McLeish, T. C. B.; Likhtman, A. E. J . Rheol 2001, 45, 539.

(8) Graham, R. S.; Likhtman, A. E.; McLeish, T. C. B.; Milner, S. T. J. Rheol. 2003, 47, 1171.

(9) Boué, F.; Nierlich, M.; J annink, G.; Ball, R. C. J . Phys. (Paris) 1982, 43, 137. Boué, F.; Nierlich, M.; J annink, G.; Ball, R. C. J . Phys., Lett. 1982, 43, L585. Boué, F.; Nierlich, M.; J annink, G.; Ball, R. C. J. Phys., Lett. 1982, 43, L593.

(10) Boué, F. Adv. Polym. Sci. 1987, 82, 47.

(11) Boué, F.; Bastide, J .; Buzier, M.; Lapp, A.; Herz, J .; Vilgis, T. A. Colloid Polym. Sci. 1991, 269, 195.

(12) Mendes, E.; Oeser, R.; Hayes, C.; Boué, F.; Bastide, J. Macromolecules 1996, 29, 5574.

(13) Hayes, C.; Bokobza, L.; Boué, F.; Mendes, E.; Monnerie, L. Macromolecules 1996, 29, 5036.

(14) Rouf, C.; Bastide, J .; Pujol, J .; Schosseler, F.; Munch, J . P. Phys. Rev. Lett. 1994, 73, 830.

(15) Blanchard, A.; et al. Manuscript in preparation.

(16) Bent, J .; Hutchings, L. R.; Richards, R. W.; Gough, T.; Spares, R.; Coates, P. D.; Grillo, I.; Harlen, O. G.; Read, D. J .; Graham, R. S.; Likhtman, A. E.; Groves, D. J .; Nicholson, T. M.; McLeish, T. C. B. Science 2003, 301, 1691.

(17) McLeish, T. C. B.; Larson, R. G. J. Rheol. 1998, 42, 81.

(18) Inkson, N. J .; McLeish, T. C. B.; Harlen, O. G.; Groves, D. J . . Rheol. 1999, 43, 873

(19) Blackwell, R. J .; McLeish, T. C. B.; Harlen, O. G. J . Rheol 2000, 44, 121.

(20) McLeish, T. C. B. Macromolecules 1988, 21, 1062.

(21) Bick, D. K.; McLeish, T. C. B. Phys. Rev. Lett. 1996, 76, 2587.

(22) McLeish, T. C. B.; Allgaier, J .; Bick, D. K.; Bishko, G.; Biswas, P.; Blackwell, R.; Blottière, B.; Clarke, N.; Gibbs, B.; Groves, D. J .; Hakiki, A.; Heenan, R.; J ohnson, J. M.; Kant, R.; Read, D. J .; Young, R. N. Macromolecules 1999, 32, 6734.

(23) Heinrich, M.; Pyckhout-Hintzen, W.; Allgaier, J .; Richter, D.; Straube, E.; Read, D. J .; McLeish, T. C. B.; Groves, D. J .; Blackwell, R. J .; Wiedenmann, A. Macromol ecules 2002, 35, 6650.

(24) McLeish, T. C. B. Polym. Commun. 1989, 30, 4.

(25) Read, D. J . Eur. Phys. J . B 1999, 12, 431.

(26) Read, D. J . Macromol ecules 2004, 37, 5065

(27) Perny, S.; Allgaier, J .; Cho, D.; Lee, W.; Chang, T. Macromolecules 2001, 34, 5408.

(28) Ferry, J . D. Viscoelastic Properties of Polymers, 3rd ed.; J Wiley \& Sons: New York, 1980.

(29) Fetters, L. J .; Lohse, D. J .; Richter, D.; Witten, T. A.; Zirkel, A. Macromolecules 1994, 27, 4639.

(30) Westermann, S.; Urban, V.; Pyckhout-Hintzen, W.; Richter, D.; Straube, E. Macromolecules 1996, 29, 6165.

(31) Warner, M.; Edwards, S. F. J . Phys. A 1978, 11, 1649

(32) Milner, S. T.; McLeish, T. C. B. Macromolecules 1997, 30 2159.

(33) Colby, R. H.; Rubinstein, M. Macromol ecules 1990, 23, 2753.

(34) Heinrich, G.; Straube, E. Polym. Bull. (Berlin) 1987, 17, 255.

(35) Rubinstein, M.; Panyukov, S. Macromolecules 1997, 30, 8036

(36) Rubinstein, M.; Panyukov, S. Macromolecules 2002, 35, 6670.

(37) Mergell, B.; Everaers, R. Macromolecules 2001, 34, 5675.

(38) Leibler, L.; Benoit, H. Polymer 1981, 22, 195.

(39) Westermann, S.; Wischnewski, A.; Pyckhout-Hintzen, W.; Richter, D.; Straube, E.; Farago, B. Manuscript in preparation.

(40) Watanabe, H.; Matsumiya, Y.; Inoue, T. Macromolecules 2002, 35, 2339.

(41) McLeish, T. C. B. J . Rheol. 2003, 47, 177.

MA0304372 